\title{
Abbreviations and Symbols
}

AEO

ANET

APAW

ASAE

$\mathrm{B} A R$

Berlin

Festschrift

$B I F A O$

Bonnet, $R \ddot{A} R G$

Borchardt,

Statuen

Bresciani, Letteratura

Brugsch, Thesaurus

BrunnerTraut, Märchen

$C d \dot{E}$ Černý, Copt. Dic.

CRAIBL

Erichsen, Lesestücke

Esna
A. H. Gardiner. Ancient Egyptian Onomastica. 3 vols. Oxford, 1947.

Ancient Near Eastern Texts Relating to the Old Testament, ed. J. B. Pritchard. Princeton, 1950; 3d ed., 1969. Abhandlungen der Preussischen Akademie der Wissenschaften.

Annales du Service des Antiquités de l'Égypte.

J. H. Breasted. Ancient Records of Egypt. 5 vols. Chicago, 1906-1907. Reprint, 1962.

Festschrift zum 150 jährigen Bestehen des Berliner Ägyptischen Museums. Staatliche Museen zu Berlin. Mitteilungen aus der ägyptischen Sammlung, 8. Berlin, 1974.

Bulletin de l'Institut Français d'Archéologie Orientale du Caire.

H. Bonnet. Reallexikon der ägyptischen Religionsgeschichte. Berlin, 1952.

L. Borchardt. Statuen und Statuetten von Königen und Privatleuten im Museum von Kairo. Vol. II. Catalogue général ... du Musée du Caire. Berlin, 1925.

E. Bresciani, Letteratura e poesia dell' antico Egitto. Turin, 1969.

H. K. Brugsch. Thesaurus Inscriptionum Aegyptiacarum. 6 parts. Leipzig, 1883-1891. Reprint, 1968.

E. Brunner-Traut. Altägyptische Märchen. Dusseldorf and Cologne, 1963. 2d ed., 1965.

Chronique d'Égypte.

J. Černý. Coptic Etymological Dictionary. Cambridge, 1976.

Comptes rendus de l'Académie des Inscriptions et Belleslettres.

W. Erichsen. Demotische Lesestücke. Leipzig, 1937.

S. Sauneron. Esna. 5 vols. Cairo, 1959-1963. 
Glossar

Harris,

Minerals

JAOS

JARCE

$J E A$

JNES

Kees,

Priester

Kees,

Priestertum

Kitchen, Inscriptions

Kitchen,

Third

Intermediate

Leclant, Recherches

Lefebvre, Romans

Legrain,

Statues

Mariette, Mon. div.

MDIK

Mélanges

Maspero I MIO

Miscellanea

Gregoriana

Montet, Géographie MPON
W. Erichsen. Demotisches Glossar. Copenhagen, 1954. J. R. Harris. Lexicographical Studies in Ancient Egyptian Minerals. Akademie der Wissenschaften, Berlin. Institut für Orientforschung. Veröffentlichung, 54. Berlin, 1961.

Journal of the American Oriental Society.

Journal of the American Research Center in Egypt.

Journal of Egyptian Archaeology.

Journal of Near Eastern Studies.

H. Kees. Die Hohenpriester des Amun von Karnak von Herihor bis zum Ende der Äthiopenzeit. Probleme der Ägyptologie, 4. Leiden, 1964.

H. Kees. Das Priestertum im ägyptischen Staat. Probleme der Ägyptologie, 1. Leiden, 1953. Nachträge, 1958. K. A. Kitchen. Ramesside Inscriptions, Historical and Biographical. Oxford, 1968-.

K. A. Kitchen. The Third Intermediate Period in Egypt. Warminster, 1973.

J. Leclant. Recherches sur les monuments thébains de la $X X V e$ dynastie dite éthiopienne. Institut Français d'Archéologie Orientale du Caire. Bibliothèque d'étude, 36. Cairo, 1965.

G. Lefebvre. Romans et contes égyptiens de l'époque pharaonique. Paris, 1949.

G. Legrain. Statues et statuettes de rois et de particuliers. Vol. III. Catalogue général ... du Musée du Caire. Cairo, 1914.

A. Mariette. Monuments divers recueillis en Égypte et en Nubie. Paris, 1872-1889.

Mitteilungen des deutschen archäologischen Instituts, $A b$ teilung Kairo.

Orient Ancien. Institut Français d'Archéologie Orientale du Caire. Mémoires, 66. Cairo, 1934-1961.

Akademie der Wissenschaften, Berlin. Mitteilungen des Instituts für Orientforschung.

Miscellanea Gregoriana: Raccolta di scritti pubblicati nel i centenario dalla fondazione del Pont. Museo Egizio. Rome, 1941.

P. Montet. Géographie de l'Égypte ancienne. 2 vols. Paris, 1957-1961.

Mitteilungen aus der Papyrussammlung der österreichischen Nationalbibliothek (Papyrus Erzherzog Rainer). 
Müller, Liebespoesie

W. M. Müller. Die Liebespoesie der alten Ägypter. LeipMythus zig, 1899.

Mythus

W. Spiegelberg. Der ägyptische Mythus vom Sonnenauge (Der Papyrus der Tierfabeln "Kuf") nach dem Leidener Demotischen Papyrus I 384. Strassburg, 1917.

NGWG Nachrichten von der Gesellschaft der Wissenschaften zu Göttingen.

$O L Z \quad$ Orientalistische Literaturzeitung.

OMRO Leiden. Rijksmuseum van Oudheden. Oudheidkundige mededeelingen.

Otto Gedenk- Fragen an die altägyptische Literatur; Studien zum Geschrift

Otto,

Inschriften

Pierret,

Recueil

P. Krall

$P M$ denken an Eberhard Otto. Wiesbaden, 1977.

E. Otto. Die biographischen Inschriften der ägyptischen Spätzeit. Probleme der Ägyptologie, 2. Leiden, 1954. P. Pierret. Recueil d'inscriptions inédites du Musée Égyptien du Louvre. 2 vols. Paris, 1874-1878.

E. Bresciani. Der Kampf um den Panzer des Inaros (Papyrus Krall). MPON, n.s. 8. Vienna, 1964.

Topographical Bibliography of Ancient Egyptian Hieroglyphic Texts, Reliefs, and Paintings, by B. Porter and R. L. B. Moss. 7 vols. Oxford, 1927-1951. 2d ed., 1960-

$R B$

Revue Biblique.

$R d E$

Roeder, Revue d'Égyptologie.

Götterwelt $R T$

G. Roeder. Die ägyptische Götterwelt. Die ägyptische Religion in Texten und Bildern, 1. Zurich 1959. Recueil de travaux relatifs à la philologie et à l'archéologie égyptiennes et assyriennes.

Sachau Festschrift Eduard Sachau, ed. G. Weil. Berlin, 1915. $S A K$ Studien zur altägyptischen Kultur.

Schott, Liebeslieder

Studi

Rosellini

Untersuchungen Urk. II

S. Schott. Altägyptische Liebeslieder, mit Märchen und Liebesgeschichten. Zurich, 1950.

Studi in memoria di Ippolito Rosellini. 2 vols. Pisa, 19491955.

Untersuchungen zur Geschichte und Altertumskunde Ägyptens.

Urkunden des ägyptischen Altertums, Abteilung II: Hieroglyphische Urkunden der griechisch-römischen Zeit, ed. K. Sethe. Leipzig, 1904.

Urk. III

Urkunden des ägyptischen Altertums, Abteilung III: Urkunden der älteren Äthiopenkönige, ed. H. Schäfer. Leipzig, 1905. 
Urk. VIII

Urkunden des ägyptischen Altertums, Abteilung VIII: Thebanische Tempelinschriften aus griechisch-römischer Zeit, ed. K. Sethe and O. Firchow. Berlin, 1957.

Vercoutter, J. Vercoutter. Textes biographiques du Sérapéum de MemTextes biogr.

Volten,

Traumdeutung

Wb. phis. Bibliothèque de l'École des Hautes Études, IVe section, 316. Paris, 1962.

A. Volten. Demotische Traumdeutung. Analecta Aegyptiaca, 3. Copenhagen, 1942.

Wörterbuch der ägyptischen Sprache, ed. A. Erman and H. Grapow. 7 vols. Leipzig, 1926-1963.

Westendorf, W. Westendorf. Koptisches Handwörterbuch. HeidelKopt. Hw. berg, 1965-1977.

$Z \ddot{A} S$ Zeitschrift für ägyptische Sprache und Altertumskunde.

Half brackets $\lceil$ Tare used instead of question marks to signify doubt.

Square brackets [ ] enclose restorations.

Angle brackets ( ) enclose words omitted by the scribe.

Parentheses ( ) enclose additions in the English translation.

A row of three dots ... indicates the omission in the English translation of one or two words. A row of six dots ..... . indicates a longer omission.

A row of three dashes - - - indicates a short lacuna in the text. A row of six dashes - - - - - indicates a lengthy lacuna. 